Editorial

\title{
La producción científica en la UTP: una mirada a su evolución desde Scopus
}

Es un hecho indiscutible que la producción científica en la región (eje cafetero) y departamento de Risaralda tiene por líder a la Universidad Tecnológica de Pereira (1). Los resultados de la medición de grupos e investigadores, donde claramente se observó un incremento significativo en el número de ellos reconocidos y clasificados, así lo refleja.

Sin embargo, es importante observar entre otras cosas, el estado de la producción de documentos científicos en revistas indizadas en grandes bases de datos internacionales, una de ellas, además muy tomada en cuenta en la actualidad, Scopus.

Scopus, como ha sido comentado previamente (2), ésta es una base de datos de gran relevancia internacional, tal como Science Citation Index (SCI) (por cierto aún no suscrita por la UTP), no solamente recopila información bibliográfica, sino que analiza el comportamiento de las citas recibidas por las revistas y con base a esto permite generar una gran cantidad de indicadores bibliométricos y citacionales, como el h index, el SJR y el SNIP, entre otros; que permiten evaluar el desempeño de las revistas, pero también de autores, grupos de investigación, instituciones, países e incluso regiones del mundo (2).

Al revisar esta base de datos, se puede apreciar que la UTP ya cuenta en su producción con más de 700 artículos (716 al 30 de junio de 2015), donde desde el año 2009 se publican más de 50 artículos por año (Figura 1), pero desde 2013 se han publicado más de 100 por año (102 en 2013 y 129 en 2014) (Figura 1). Al ritmo actual, donde hasta el 30 de junio de 2015 se registran 60 artículos, es fácil anticipar que el año cerrará también con más de 100 artículos (Figura 1).

Este ritmo, debe mantenerse, pero sobre todo incrementarse, ya que si bien, como ha sido reportado (2), las facultades relacionadas con la ingeniería han sido líderes, han emergido otras como ciencias de la salud y en particular dentro de esta medicina, las cuales representan en total histórico acumulado de la institución 36,6\% y 32,1\% respectivamente (Figura 2), totalizando casi el $70 \%$ de toda la producción de la universidad. Sin embargo si solo se observan los artículos de 2014, 44,2\% de ellos fueron de medicina y 28,7\% de las ingenierías.

En cuanto a la cooperación internacional, si bien esta ha mejorado, aún es muy importante incrementar dicha actividad. El país con el cuál históricamente la UTP ha tenido mayor cooperación en la producción científica es España, pero solo alcanza un 13\% y de allí siguen otros países como Brasil (8\%) y Reino Unido (6\%) (Figura 3).

Llama la atención que la producción nacional es más baja que la internacional, otro elemento que debería promoverse más de acuerdo a los resultados del presente análisis (Figura 4). Como es de esperar, la principal institución del país es a su vez la principal con la cual se ha generado dicha cooperación, la Universidad Nacional de Colombia, seguido por la Universidad del Valle, la Universidad Industrial de Santander y la Universidad de Los Andes. De quinto lugar llama la atención que aún en 2015, dos años entrar en inactividad, la Oficina de Investigación Científica de la Cooperativa de Entidades de Salud de Risaralda (COODESURIS), sea la quinta institución nacional con la cual históricamente se han producido mayor cantidad de artículos en Scopus (Figura 4). 
Figura 1. Evolución de la Producción científica de la UTP registrada en la base de datos Scopus, 1982-2015 (al 30 de junio).

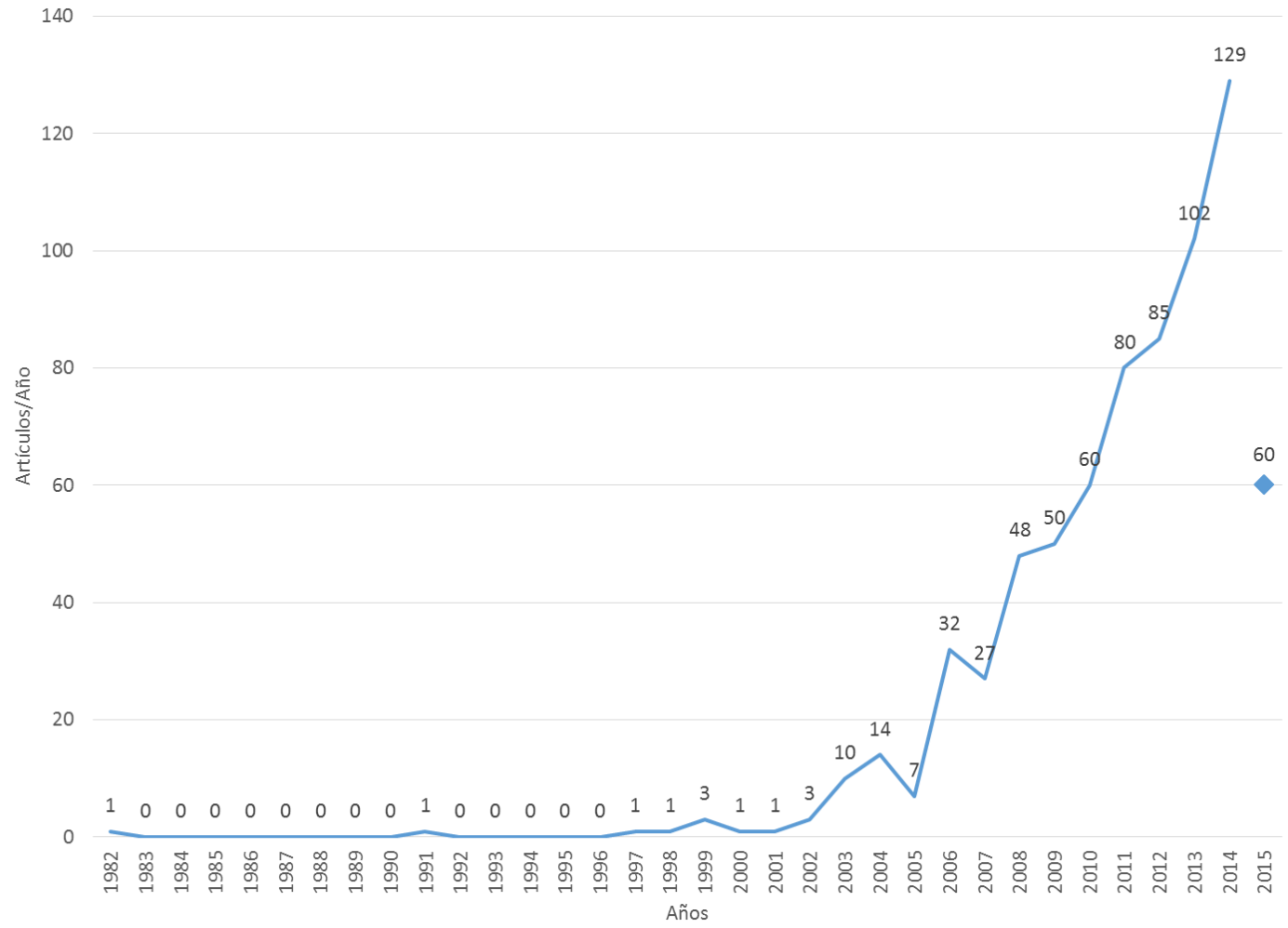

Figura 2. Distribución por áreas de la Producción científica de la UTP registrada en la base de datos Scopus, 1982-2015 (al 30 de junio).

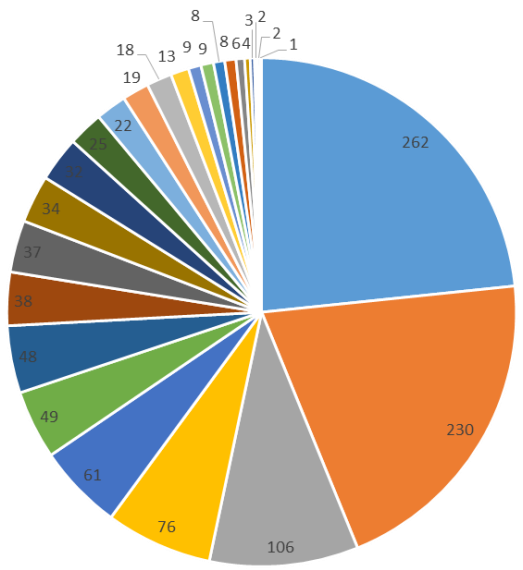

\begin{tabular}{|c|c|c|}
\hline - Engineering & - Medicine & = Computer Science \\
\hline = Energy & - Physics and Astronomy & - Agricultural and Biological Sciences \\
\hline - Biochemistry, Genetics and Molecular Biology & - Mathematics & - Environmental Science \\
\hline - Pharmacology, Toxicology and Pharmaceutics & - Materials Science & - Social Sciences \\
\hline - Chemical Engineering & - Immunology and Microbiology & = Chemistry \\
\hline Arts and Humanities & - Business, Management and Accounting & - Earth and Planetary Sciences \\
\hline - Health Professions & - Multidisciplinary & - Neuroscience \\
\hline - Economics, Econometrics and Finance & - Veterinary & - Psychology \\
\hline - Undefined & Inecision Sciences & \\
\hline
\end{tabular}


Figura 3. Cooperación internacional (\%) en la Producción científica de la UTP registrada en la base de datos Scopus, 1982-2015 (al 30 de junio).

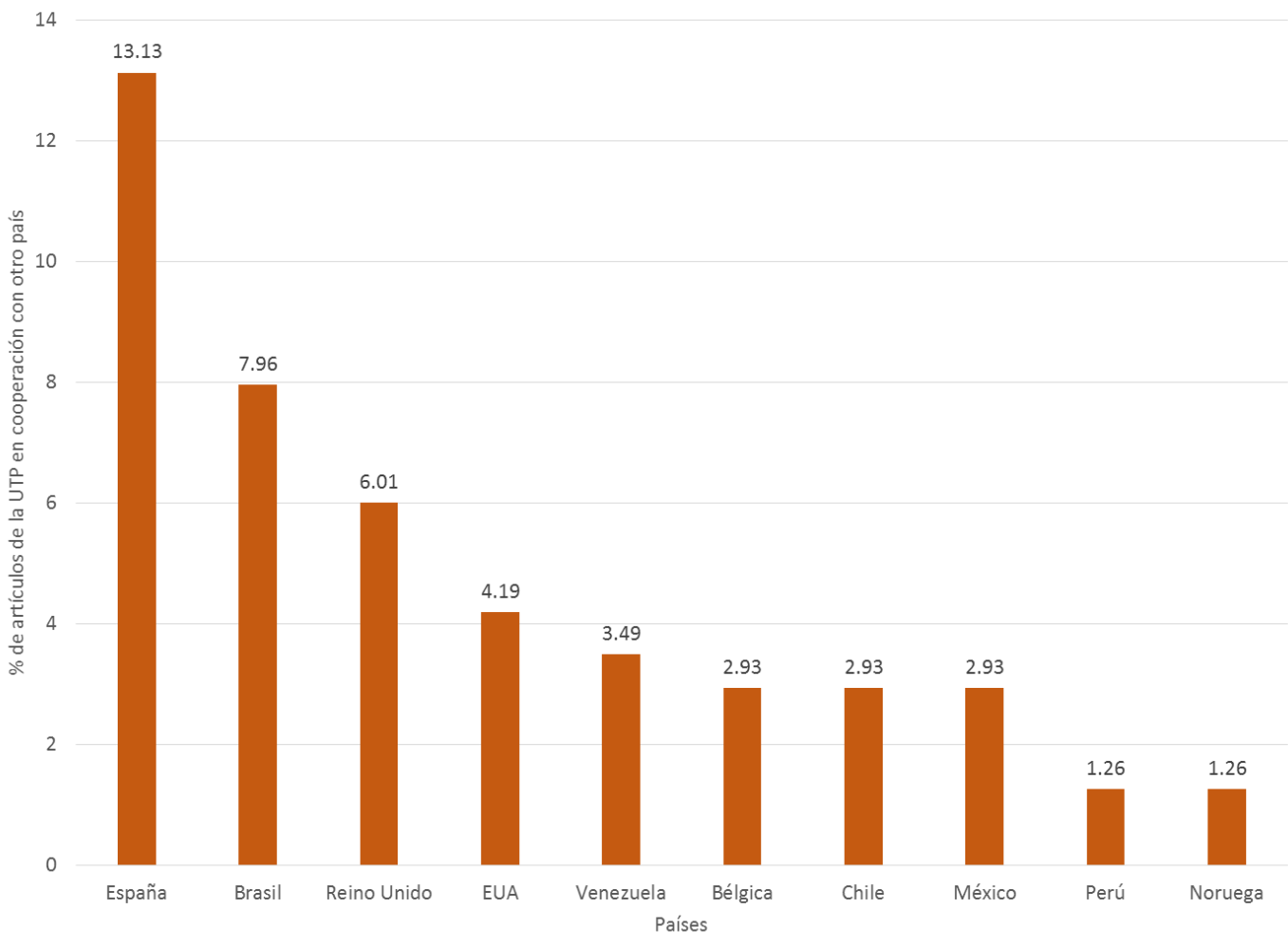

Figura 4. Cooperación nacional (número de artículos) en la Producción científica de la UTP registrada en la base de datos Scopus, 1982-2015 (al 30 de junio) (primeras 10 instituciones).

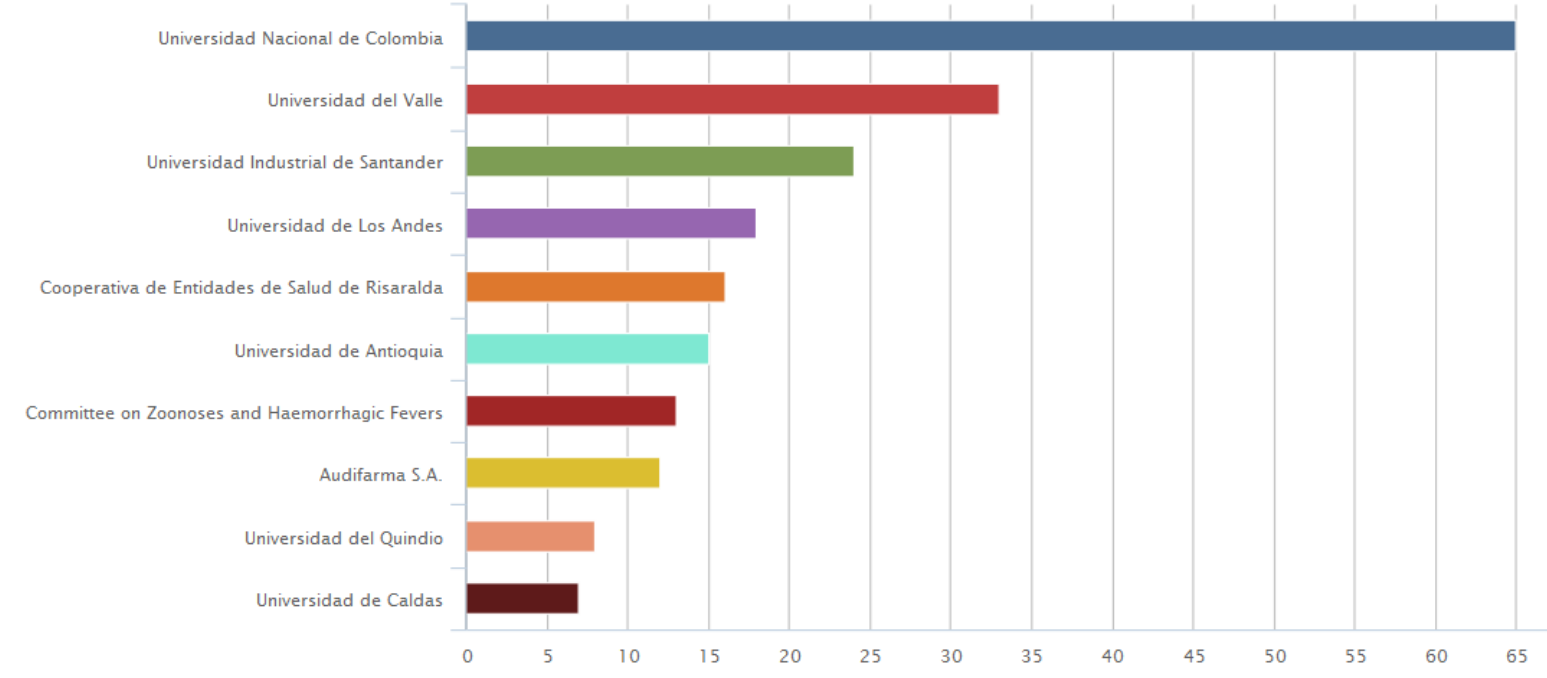

Finalmente es importante comentar acerca del impacto citacional que tiene la producción científica de la institución en la base de datos Scopus. Los 716 trabajos publicados han recibido 2378 citas, es decir cada artículo ha recibido en promedio 3,3 citas, siendo el artículo más citado el del profesor Ramón Alfonso Gallego, "Optimal capacitor placement in radial 
distribution networks" en conjunto con autores de Brasil, publicado en IEEE Transactions on Power Systems en Noviembre 2001 (Vol. 16, No. 4, 630-637), docente que cuenta a su vez con un $\mathrm{H}$ index de 9. El H index de la UTP es de 21, lo cual quiere decir que se registran en Scopus 21 artículos de la institución que cuentan con al menos 21 citas o más cada uno.

El número mencionado de citas muestra un claro incremento por año, desde el año 2000 hasta 2015, que indica un interés cada vez mayor en la comunidad científica nacional e internacional, por la producción de la UTP (Figura 5).

Figura 5. Evolución de las citas recibidas por la producción científica de la UTP registrada en la base de datos Scopus, 2000-2015 (al 30 de junio). 600

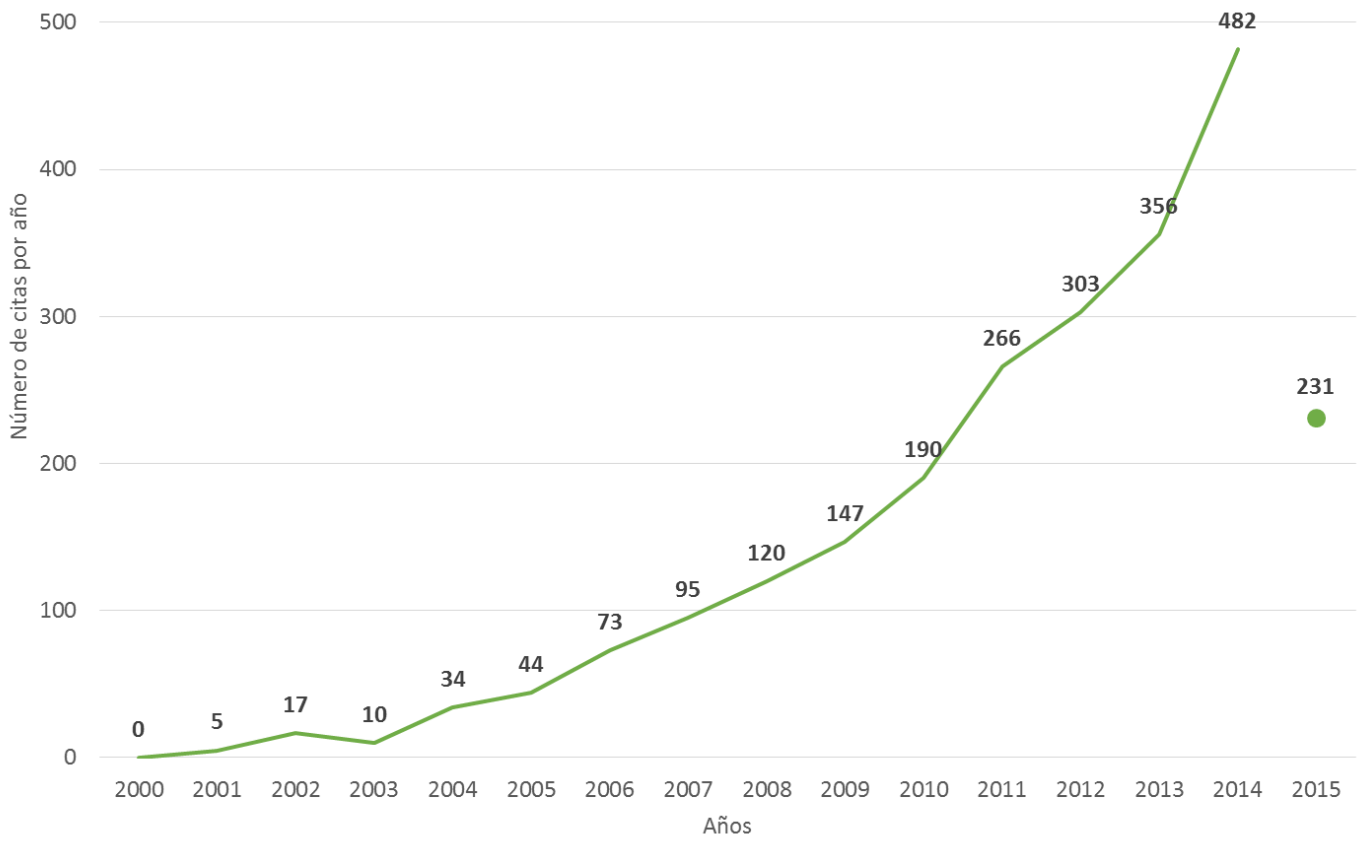

La UTP ha crecido en gran forma en investigación, sin embargo debe seguir profundizando sus procesos, aumentando el número de grupos reconocidos y clasificados por Colciencias, la proporción de ellos que son "A1", mejorar sus revistas indizadas (posicionándolas en mejores bases de datos), pero también manteniendo firme el incremento en la producción científica de artículos, para lo cual la formación continua en redacción científica (3), incluida desde pregrado (4), es un elemento de vital importancia, como también ha sido manifestado, para que pueda entrar y mantenerse entre las primeras 10 instituciones del país en producción científica en la base de datos Scopus.

\section{Alfonso J. Rodríguez-Morales}

Editor Asesor, Revista Scientia et Technica, Docente Transitorio, Universidad Tecnológica de Pereira, Pereira, Risaralda, Colombia. e-mail: arodriguezm@utp.edu.co 


\section{Referencias}

1. Rodríguez-Morales AJ, Rendón MA. Situación de la investigación y publicación científica en Colombia y en el departamento de Risaralda: Revisión de indicadores en el contexto de la creación de la Oficina de Investigación Científica de COODESURIS. Revista Médica de Risaralda 2012; 18(1):72-82.

2. Rodríguez-Morales AJ. Scopus y su importancia actual en la publicación científica colombiana. Scientia et Technica 2013;18(4):iii-v.

3. Rodriguez-Morales AJ, López Quintero JG. El Nuevo Diplomado de Investigación y Publicación Científica de la UTP. Revista Médica de Risaralda 2013 Abr;19(1):97-98.

4. Huamaní C, Mayta-Tristán P, Rodríguez-Morales AJ. Publicar desde Pregrado. Interciencia 2008 Nov; $33(11): 785$ 